\title{
Diseño de un módulo electrónico para la crianza automatizada de peces mediante modelamiento matemático multiparamétrico que simule las condiciones básicas necesarias para la crianza, en estanques artificiales en función de parámetros fisicoquímicos
}

\section{Electronic module design for automatic fish breeding through multiparametric mathematical modeling that simulates necessary basic conditions for breeding in artificial ponds according to physico-chemical parameters}

\author{
Hernán Díaz-Lopez ${ }^{1}$, Yezid Vargas-Gómez ${ }^{2}$
}

\begin{abstract}
${ }^{1}$ Grupo de Investigación en Tecnologías Socioeconómicas e Ingenierías (Gitsein), Facultad de Ciencias Naturales e Ingenierías, Programa de Electrónica Industrial, Unidades Tecnológicas de Santander, Colombia. Email: hdiaz@ correo.uts.edu.co

${ }^{2}$ Grupo de Investigación en Tecnologías Socioeconómicas e Ingenierías (Gitsein), Facultad de Ciencias Naturales e Ingenierías, Programa de Electrónica Industrial, Unidades Tecnológicas de Santander, Colombia. Email: yvargas@ correo.uts.edu.co
\end{abstract}

Recibido: septiembre 28, 2017. Aceptado: marzo 19, 2018. Versión final: mayo 11, 2018.

\begin{abstract}
Resumen
La acuicultura es una de las actividades que, en el sector productivo, ha tenido un crecimiento económico en el ámbito nacional. Esta depende del manejo que se le pueda brindar al cuerpo de agua, lo cual requiere la atención de ciertos parámetros fisicoquímicos como temperatura, oxígeno disuelto, $\mathrm{pH}$, entre otros, para obtener el éxito de la producción. En este trabajo se muestra el estudio de varios modelos matemáticos en los que se toma la calidad de agua como caso de estudio, con el propósito de simplificarlos, usando tres metodologías a través de herramientas computacionales, así como de las relaciones o evolución temporal de cada variable, expresadas a través de relaciones matemáticas correspondientes del mundo real (relaciones tecnológicas, leyes físicas, restricciones del mercado, etc.) estimando el comportamiento del proceso para determinadas condiciones.
\end{abstract}

En primera instancia se definen los parámetros para la caracterización, tales como el régimen de alimentación, biomasa, alcalinidad, aireación, efectos fotosintéticos, entre otros factores físicos, químicos y biológicos de fácil medición, en una secuencia de casos particulares. Con esto se adapta el modelo matemático tomando elementos desde su codificación en ecuaciones, las cuales permiten hacer un análisis y encontrar una expresión para su concentración en estado estacionario; mediante la relación de estas cantidades con la concentración máxima admisible, que se espera poder condensar en un "único" modelo multifactorial que caracterice todo el proceso, buscando mantener ciertos parámetros considerados como críticos, dentro de unos límites aceptables. Esto, basados en modelos estandarizados, incorporando las modificaciones o mejoras que cada uno de ellos aporta, mediante el estudio previo que ha pretendido integrar todo el proceso en el interior de estanques artificiales de geomembrana, implementando herramientas de monitoreo que posibilitan el manejo estadístico, registrar cambios en los patrones, además de poder generar reportes históricos e información importante del proceso. 
Se realiza la identificación en sistemas reales haciendo uso de la herramienta "Control System Toolbox", de Matlab, a través de una versión institucional; esto permite la obtención de una cantidad de datos significativos con suficiente información de la dinámica del sistema, validando varios modelos, reduciendo la solución a la "mínima expresión". Adicionalmente, se diseña una interfaz que facilita el ingreso de parámetros, simula diferentes escenarios de cultivo o condiciones iniciales del sistema para la estimación de las múltiples variables en un número reducido. La interfaz permite, de igual manera, determinar el número máximo de la población cultivada que el ambiente puede soportar en un periodo de tiempo, que condescienda en la adecuada operación de proyectos piscícolas de forma continua, sin afectar la salud de los peces, debido en gran medida a la falta de un instrumento para controlar la calidad del agua del proceso; de modo que minimice el impacto ambiental, mejore los beneficios comerciales, atendiendo especialmente a aquellos aspectos que más influyen en el cultivo comercial, cumpliendo con las recomendaciones sobre estabulación de los peces. El proyecto ha atendido a la necesidad de una serie de procedimientos, observaciones, así como recomendaciones al respecto.

Palabras clave: factores físicos; factores químicos; herramientas computacionales; modelo matemático; parámetros en la calidad del agua; $\mathrm{pH}$; piscicultura; oxígeno disuelto; temperatura.

\begin{abstract}
Aquaculture is one of the activities that has had economic growth in the productive sector at a national level. This activity depends on the management that can be given to the body of water, which requires attention on certain physicochemical parameters such as temperature, dissolved oxygen, $\mathrm{pH}$, among others to obtain production success. In this work we show the study of several mathematical models where water quality is taken as a case study, with the purpose of simplifying them, maintaining the maximum of the model using three methodologies through computational tools, as well as relations or temporal evolution of each variable, expressed through corresponding mathematical relations of the real world (technological relations, physical laws, market restrictions, etc.) estimating the behavior of the process for certain conditions.
\end{abstract}

In the first instance, the parameters for the characterization such as feed regime, biomass, alkalinity, aeration, photosynthetic effects, among other physical, chemical and biological factors of easier measurement are defined in a sequence of particular cases. With this, the mathematical model is adapted by taking elements from their coding in equations, which allow an analysis of inputs / outputs to find an expression for their concentration in steady state by the ratio of these quantities to the maximum admissible concentration, which is expected, to be condensed into a "single" multifactorial model that characterizes the whole process, seeking to maintain certain parameters considered as critical, within acceptable limits. All this, based on standardized models incorporating the modifications or improvements that each one of them contributes with and through the previous study that has tried to integrate all the process inside artificial geomembrane ponds, implementing monitoring tools that allow for statistical management, registration of changes in the patterns, besides being able to generate historical reports, and important information of the process.

The identification in real systems is made using the MATLAB Toolbox, obtaining a significant amount of data to ensure sufficient information of the dynamics of the system, validating several models, reducing the solution to "minimum expression". Additionally, an interface that facilitates the input of parameters is designed; it simulates different crop scenarios or initial conditions of the system for the estimation of the multiple variables in a reduced number of these. Likewise, the interface allows to determine the maximum number of cultivated population that the environment can withstand in a period of time, that condenses in the proper operation of fishery projects in a continuous way without affecting the health of the fish, this due to a great extent to the lack of a control instrument to help control the water quality of the process; so as to minimize the environmental impact, improve the commercial benefits, paying special attention to those aspects that most influence the commercial culture, complying with the recommendations on fish stocking; This work being a starting point, presenting a series of procedures, observations, and recommendations.

Keywords: physical factors; chemical factors; computational tools; mathematical model; parameters in water quality; $\mathrm{pH}$; fish farming; dissolved oxygen; temperature. 
Diseño de un módulo electrónico para la crianza automatizada de peces mediante modelamiento matemático multiparamétrico que simule las condiciones básicas necesarias para la crianza, en estanques artificiales en función de parámetros fisicoquímicos

\section{Introducción}

La proyección en la demanda de carne de peces debe estar sujeta a mejoramientos tecnológicos que permitan optimizar sus procesos al utilizar de forma eficiente sus recursos, para producir una mayor cantidad de peces de atractivo valor comercial, y brindar ventajas como reducción de costes logísticos, reducción en el nivel de mortandad, mayores utilidades, mayor rentabilidad, monitoreo automático permanente para un mejor control, bajos costos de mantenimiento, obtención de mayor valor agregado, entre otros.

El costo de inversión tecnológico, además de su consumo energético, hace que a los productores no les sea rentable realizar estas prácticas de una forma más adecuada. Con este trabajo se busca entregar una herramienta integral, que anticipadamente estima de manera correlacionada estos parámetros representativos en el cultivo de peces, al análisis posterior y sistematizadamente en tiempo real, si se encuentran en normal estado, lo que lleva a normalizar o colocar en aviso al productor.

Parte de la metodología empleada consiste en el estudio de algunos de los modelos utilizados sobre algunos tipos de cuerpos de agua ([6], [22]) en los que la alimentación se realiza de forma artificial ([4], [23]), ya que en Colombia las pérdidas que genera este método en el cultivo de peces es del $40 \%$ de la producción [23], debido en gran medida al deterioro de la calidad del agua ([8], [24]).

Para ello se tienen en cuenta los modelos más utilizados para la estimación de la capacidad de carga en el caso particular de piscicultura intensiva, propuestos por ([19], [6], [3], [9]). A la par, el modelo propuesto por [25] para la estimación de la concentración de nutrientes en el agua, en el que se correlacionan las concentraciones de varias variables con indicadores de cambios en la transparencia, entre otros. Algunos estudios (Schindler, 1974) han confirmado al nitrógeno además del fósforo, como las principales limitantes en sistemas acuáticos ([26], [27]). Igualmente, [18] quienes definieron una serie de parámetros físico-ambientales de fácil medición, para hallar una expresión para la concentración de fósforo disuelto en estado estacionario ([18], [13]).

Así mismo, el conjunto de modelos matemáticos Activated Sludge Model, que sirve para describir sistemas complejos u optimizar el tratamiento de aguas, tales como el ASM1 (mejor predicción del nitrógeno y el fósforo), ASM2, ASM3, entre otros. El Constructed Wetlands Model 1 (CWM1), que sirve para describir los procesos de degradación de la materia orgánica y el nitrógeno, que se encarga de describir procesos aeróbicos y anóxicos, considerando otros procesos tales como la influencia de las plantas, el transporte de partículas (clogging), la aireación física, etc.

El modelo River Water Quality Model 1 (RWQM1) diseñado en su inicio para modelar procesos en aguas fluviales, así como para empezar con el estudio exhaustivo de lagunas de alta carga, que incorpora la valoración de las microalgas e incluye tanto procesos físicos como químicos, para caracterizar el carbono, oxígeno, nitrógeno y el fósforo. Asimismo, el Lacat (Lagos Cálidos Tropicales), el cual es un modelo estadístico que permite evaluar la probabilidad de eutroficación, considerando la concentración de fósforo como elemento determinante, además de estimar su concentración.

Finalmente [4], quien aplicó el modelo de Dillon y Rigler, en diferentes de cuerpos de agua ([20], [13]), probó [28] que estos modelos mejoran la capacidad predictiva, al optimizar la productividad de los proyectos piscícolas.

De esta manera, se expone un modelo teniendo en cuenta dichos referentes. Es por esto que se diseña una interfaz que facilita el ingreso de los parámetros o condiciones iniciales para la simulación de diferentes escenarios, basado en elementos variables adimensionales, con relaciones entre ellos, expresados a través de funciones matemáticas, que permiten extraer características que hacen posible experimentar o estimar con la calidad del agua en estanques piscícolas artificiales.

Para ello, en primera instancia, se definen los parámetros que se emplean para su caracterización, extraídos de la revisión bibliográfica sobre los distintos métodos para el tratamiento del agua. De ahí se desarrollaron los modelos matemáticos encargados de describir los procesos existentes en este tipo de sistemas, a partir de un análisis de entradas salidas haciendo uso de Matlab, con la intención de proponer un instrumento que ayude a controlar la calidad del agua en estos procesos de cultivo industrial, de modo que se minimice el impacto, cumpliendo las recomendaciones sobre la estabulación de los peces, al tiempo que se mejoran los beneficios comerciales.

\section{Métodos}

El sistema se estudia a partir de la metodología de Law y Kelton, donde se experimenta con el sistema físico, además de uno o varios modelos matemáticos del sistema, que han permitido llegar a una solución analítica, a partir de los resultados obtenidos en simulación, mediante el análisis, comparación o 
256 RUIIISTIS UIS

verificación finalmente, con los datos registrados de la operación del sistema actual en el dominio del tiempo

\subsection{Parámetros en estudio empleados en la modelación}

Las características del agua en un cultivo de peces se ven afectadas por factores químicos tales como oxígeno disuelto (OD), dióxido de carbono (CO2), potencial de hidrogeno $(\mathrm{pH})$, nutrientes, salinidad, sulfuro de hidrógeno (H2S), metales pesados. Asimismo, factores físicos como la temperatura, color, transparencia, turbidez, volumen de agua, caudal, recambio de agua, densidad de siembra, según se especifican a continuación.

Demanda Biológica de Oxígeno (DBO): Es la cantidad de oxígeno consumida por los microorganismos presentes en el agua para estabilizar la materia orgánica, en un cierto tiempo a una determinada temperatura. La estabilización se obtiene con la transformación de la materia orgánica en hidrógeno y dióxido de carbono. $\mathrm{Si}$ hay desechos orgánicos en el agua, habrá una cantidad importante de bacterias descomponiendo este desecho.

Oxígeno Disuelto (OD): Indicador de qué tan contaminada está el agua y del soporte que puede darse a la vida animal y vegetal. Generalmente, un nivel más alto indica agua de mejor calidad.

Según [2] el modelo de oxígeno consta de tres términos:

a) Descomposición del agua como ecuacion (1):

$$
\frac{d C_{D B O}}{d t}=\left(\begin{array}{c}
\text { Cambio de la materia } \\
\text { orgánica con respecto } \\
\text { al tiempo }
\end{array}\right)=-K_{1} C_{D B O}=\left(\begin{array}{c}
\text { Cantidad de } \\
\text { materia orgánica } \\
\text { disponible }
\end{array}\right)
$$

CDBO es la concentración de la demanda biológica de oxígeno, y K1 es la constante cinética de reacción.

b) Desoxigenación ecuacion (2):

$$
\frac{d C_{O D}}{d t}=\left(\begin{array}{c}
\text { Cambio en la concentración } \\
\text { de la demana de oxígeno con } \\
\text { respecto al tiempo }
\end{array}\right)=-K_{1} C_{D B O}=\left(\begin{array}{c}
\text { Velocidad } \\
\text { ee desaparición de } \\
\text { materia orgánica }
\end{array}\right)
$$

COD es la concentración de la demanda química de oxígeno

c) Reaireación ecuacion (3):

$$
\frac{d C_{D}}{d t}=\left(\begin{array}{c}
\text { Cambio en la concentración } \\
\text { de la demana de oxígeno con } \\
\text { respecto al tiempo }
\end{array}\right)=
$$

$$
-K_{1}\left(C_{S}-C_{O D}\right)=\left(\begin{array}{c}
\text { Velocidad de desaparición de } \\
\text { oxígeno en el agua debido a la presencia } \\
\text { de los microorganismo }
\end{array}\right)
$$

$\mathrm{CD}$ es la deficiencia de oxígeno en el agua, y Cs es la constante de oxígeno disuelto saturado en el agua.

Según [29], la concentración de OD se expresa en términos de la deficiencia de oxígeno, de tal forma que la dinámica del sistema se representa como «Acumulación = Entrada - Salida + Desoxigenación + Reaireación», ecuacion (4):

$D t=\frac{K_{1} C_{D B O}}{K_{2}-K_{1}}\left(e^{-K_{1} t}-e^{-K_{2} t}\right)+C_{D o} e^{-K_{2} t}$

Tabla 1. Nivel de DBO u OD presente en el agua.

\begin{tabular}{|c|l|}
\hline $\begin{array}{c}\text { Nivel } \\
\text { DBO } \\
(\mathbf{p p m})\end{array}$ & \multicolumn{1}{c|}{ Calidad del agua } \\
\hline 1,0 a 2,0 & $\begin{array}{l}\text { Muy buena: el desecho orgánico en la } \\
\text { muestra de agua es casi nulo }\end{array}$ \\
\hline 3,0 a 5,0 & $\begin{array}{l}\text { Aceptable: hay materia orgánica que las } \\
\text { bacterias están descomponiendo }\end{array}$ \\
\hline $\begin{array}{c}100 \text { o } \\
\text { más }\end{array}$ & $\begin{array}{l}\text { Muy mala: contaminada, contiene } \\
\text { desecho orgánico }\end{array}$ \\
\hline
\end{tabular}

\begin{tabular}{|c|l|}
\hline $\begin{array}{c}\text { Nivel OD } \\
\text { (ppm) }\end{array}$ & \multicolumn{1}{|c|}{ Calidad del agua } \\
\hline 0,0 a 4,0 & $\begin{array}{l}\text { Mala: algunos peces empezarán a } \\
\text { disminuir }\end{array}$ \\
\hline 4,1 a 7,9 & Aceptable \\
\hline 8,0 a 12 & Buena \\
\hline 12 o más & $\begin{array}{l}\text { Repetir prueba: el agua puede } \\
\text { airearse artificialmente }\end{array}$ \\
\hline
\end{tabular}

Fuente: [2].

Constante Térmica de Crecimiento (CTC): Calculada a partir del peso por grado al día, según [30] (Ecuación. 4): Índice de Crecimiento Específico (ICE): Peso alcanzado en el periodo de estudio sobre el tiempo de seguimiento ecuación (5), siendo una medida del crecimiento medio diario [30]:

Factor de Condición (K): Densidad del estado nutricional del pez o de su grado de engorde, definido como se observa en la ecuación (6), el peso dividido por el cubo de su longitud expresada en tanto por ciento [30]:

$$
\begin{aligned}
& C T C=\frac{P_{f}{ }^{1 / 3}-P_{i}{ }^{1 / 3}}{\Sigma T^{\circ} C-\text { media diaria }}(5) \quad I C E=\frac{L n P_{f}-L n P_{i}}{t(\text { en días })}(6) \\
& K=\frac{P * 100}{L^{3}}(7)
\end{aligned}
$$


Diseño de un módulo electrónico para la crianza automatizada de peces mediante modelamiento matemático multiparamétrico que simule las condiciones básicas necesarias para la crianza, en estanques artificiales en función de parámetros fisicoquímicos

La tabla 2 describe algunos de estos indicadores condicionantes en función del comportamiento, morfología y producción de los peces, además de los parámetros en la calidad del agua asociados.

Tabla 2. Parámetros indicadores de la calidad del agua.

\begin{tabular}{|c|c|c|c|}
\hline Parámetro & $\begin{array}{c}\text { Nivel } \\
\text { adecuado }\end{array}$ & $\begin{array}{c}\text { Nivel } \\
\text { letal } \\
(1)\end{array}$ & $\begin{array}{l}\text { Cuando y donde } \\
\text { monitorear }\end{array}$ \\
\hline $\mathrm{pH}$ & 6,0 & $<5,0$ & $\begin{array}{l}\text { Una vez día a su } \\
\text { inicio. Con agua } \\
\text { verde además al } \\
\text { final de la tarde. }\end{array}$ \\
\hline $\begin{array}{r}\text { Gas } \\
\text { Carbónico } \\
\end{array}$ & $\begin{array}{l}<5 \mathrm{mg} / \mathrm{L} \\
(10 \mathrm{ppm})\end{array}$ & $\begin{array}{l}>20 \\
\mathrm{mg} / \mathrm{L}\end{array}$ & $\begin{array}{l}\text { Cada } 2 \text { a } 3 \text { días al } \\
\text { principio del día. }\end{array}$ \\
\hline $\begin{array}{r}\text { Amoniaco } \\
\text { Toxico } \\
(\mathrm{NH} 4+/ \\
\mathrm{NH} 4-\mathrm{N}) \\
\end{array}$ & $\begin{array}{c}<0,2 \mathrm{mg} / \mathrm{L} \\
(0,5 \mathrm{ppm})\end{array}$ & $\begin{array}{c}>0,6 \\
\mathrm{mg} / \mathrm{L} \\
<3 \\
\mathrm{mg} / \mathrm{L}\end{array}$ & $\begin{array}{l}\text { Una vez día junto a } \\
\text { pH. }\end{array}$ \\
\hline Alcalinidad & $\begin{array}{c}50-300 \\
\mathrm{ppm}\end{array}$ & $\begin{array}{l}<30 \\
\mathrm{mg} / \mathrm{L}\end{array}$ & $\begin{array}{l}\text { Por lo menos una } \\
\text { vez semana. }\end{array}$ \\
\hline $\begin{array}{l}\text { Nitrito } \\
\text { (NO2-) }\end{array}$ & $\begin{array}{c}<0,3 \mathrm{mg} / \mathrm{L} \\
(1 \mathrm{ppm})\end{array}$ & $\begin{array}{c}>5 \\
\mathrm{mg} / \mathrm{L}\end{array}$ & $\begin{array}{l}\text { Diariamente en el } \\
\text { agua. }\end{array}$ \\
\hline Nitrato & $<50 \mathrm{mg} / \mathrm{L}$ & $\begin{array}{l}>400 \\
\mathrm{mg} / \mathrm{L}\end{array}$ & Semanalmente. \\
\hline $\begin{array}{r}\text { Sólidos en } \\
\text { suspensión }\end{array}$ & $<20 \mathrm{mg} / \mathrm{L}$ & $\begin{array}{l}>100 \\
\mathrm{mg} / \mathrm{L}\end{array}$ & Cada 2 semanas. \\
\hline
\end{tabular}

Fuente: adaptado de [32].

(1) Dependen del tiempo de exposición, especie, además del tamaño del pez, así como de la combinación de otros parámetros: (Salinidad: Agua Dulce: 0,5 ppm, Dureza: 40 a $400 \mathrm{ppm}$. Temperatura: Máxima: $35^{\circ} \mathrm{C}$, Optima: 28 a $32^{\circ} \mathrm{C}$, Mínima: $14^{\circ} \mathrm{C}$ ).

\subsection{Modelo de Dillon - Rigler y Beveridge}

Este modelo alternativo considera la concentración de fósforo P $(\mathrm{kg} / \mathrm{m} 3)$ como el principal factor limitante de la productividad de los peces, teniendo en cuenta las siguientes consideraciones:

1. El fósforo entrante se disuelve de manera uniforme e instantánea en el agua (columna + sedimentos), por lo que se debe medir un promedio en todo el cuerpo de agua durante todo el año.

2. Sea $\mathrm{T}$ el tiempo de descarga (años), y R la fracción de fósforo entrante pérdida por acumulación en los sedimentos en la ecuación (8), se pueden determinar, si no se conocen los valores en campo [4], a partir de ajustes estadísticos a datos recogidos en diferentes cuerpos de agua, utilizando [31]:
$R=1 /[1+0.747(T-0.507)]$

2. El sistema está en estado estacionario (P y L constantes), por tanto, $\mathrm{P}$ 'sale $=\mathrm{P}$ 'entra. Teniendo en cuenta que $\mathrm{V}=\mathrm{Az}$, con $\mathrm{z}$ la profundidad media del cuerpo de agua (m); L, el flujo de entrada de fósforo al sistema $(\mathrm{kg} /(\mathrm{m} 2 * \mathrm{año}))$; $\mathrm{A}$, el área superficial del cuerpo de agua, (m2) y V, el volumen del cuerpo de agua (m3); la concentración de fósforo también proviene del alimento no ingerido, así como de los excrementos de los individuos (P excedente: Pexc).

A la par, sea Lexc el flujo de entrada del fósforo excedente (kg de fósforo/ (m2 año)) y Rpez la fracción de fósforo proveniente de los cultivos piscícolas, pérdida en los sedimentos [13]. El valor límite de Pexc se estima a partir de la concentración máxima admisible de fósforo posterior a la cosecha Pfinal, así como de la concentración de fósforo promediada en la columna de agua en momentos previos al inicio de toda actividad de piscicultura, Pinicial; por lo que [4] propuso estimar la producción de biomasa o capacidad de carga anual (CC), a partir de la razón entre la rapidez de entrada del fósforo excedente (Pexc /año) con el descargado al sistema producto de los cultivos (Pexc/Tonelada de pez producida), donde Pexc es obtenido a partir del fósforo que entra como alimento, así como del que sale como biomasa, todo esto según la ecuación (9) [15]:

$$
\begin{aligned}
C C & =\frac{P_{\text {exc }}^{\prime}}{P_{\text {exc }}}=\frac{L_{\text {exc }} A}{\frac{\left(T C A * \% P_{a l}-\% P_{\text {pez }}\right)}{100}} \\
= & \frac{\left(P_{\text {final }}-P_{\text {inicial }}\right) * V}{T\left(1-R_{\text {pez }}\right)\left(T C A * \% P_{\text {al }} \% P_{\text {pez }}\right) / 100}
\end{aligned}
$$

Donde \%Pal es el porcentaje de fósforo contenido en el alimento; \%Ppez, el de fósforo en los peces, constante al final de la cosecha, y TCA, la tasa de conversión alimenticia, definida como la eficacia de la alimentación: Alimento Suministrado $(\mathrm{kg}) /$ Aumento de la Biomasa $(\mathrm{kg})=$ Ingesta $/(\mathrm{Pf}-\mathrm{Pi})$, que comercialmente varía entre 1,4 a 3,0 [4].

\subsection{Modelo dinámico}

A fin de obtener una mayor cantidad de elementos dentro del modelo, que puedan ser sintetizados en una nueva propuesta, se considera, además, la ecuación para la Biomasa Bm: directamente proporcional al flujo de entrada de alimento IA ( $\mathrm{kg}$ alimento/día), e inversamente proporcional al factor que, de este alimento, el pez efectivamente transforma en biomasa (TCA), como se puede apreciar en la ecuación (10) [15]: 
$B m(t)=B m(0)+\frac{I_{A}}{T C A} t$

Se fija como solución de equilibrio para $\mathrm{P}$ el máximo permitido Pfinal, que se consigue en un tiempo de equilibrio teq. En este instante, la tasa de variación de $\mathrm{P}$ es nula, e IA se interpreta como el valor necesario para lograr el máximo admisible Pfinal para la concentración de $\mathrm{P}$ en estado de equilibrio, donde la capacidad de carga viene dada por $\mathrm{CC}=\mathrm{IA} / \mathrm{TCA}$.

La condición inicial $\mathrm{Bm}(0)$ está dada por bmalevino * $\mathrm{N}$, con $\mathrm{N}$, el número de alevinos que ingresan al sistema y bmalevino, el peso del alevino que se cultiva, sin tener en cuenta la mortalidad de los peces u otras variables.

Para elegir IA existen dos opciones: tomar un valor constante estimado a partir del promedio del alimento suministrado en un tiempo determinado, o tomar IA variable teniendo en cuenta las tablas de alimentación, en las cuales se sugiere que la cantidad diaria de alimento sea un porcentaje de la biomasa, es decir, IA $=\mathrm{cBm}$. Es así como para obtener la ecuación para el fósforo disuelto $\mathrm{P}$, se han utilizado además las siguientes consideraciones:

1. Entrada proveniente de otras fuentes diferentes a la piscicultura, fotros ( $\mathrm{kg}$ de fósforo/día), y del flujo de entrada del fósforo proveniente del alimento al sistema, fal (kg de fósforo/día), proporcional a IA con \% Pal.

2. Salida de fósforo dada por la suma de la descarga del cuerpo de agua y del fósforo presente en el alimento. Se puede expresar como el producto entre \%Ppez con $\mathrm{dBm} / \mathrm{dt}$, multiplicado por el factor (1 - Rpez), porque el pez solo tiene acceso al fósforo que permanece en la columna de agua. La ecuación (11) para el fósforo P se obtiene de la resta entre P'entra con P'sale:

$$
\begin{aligned}
& \frac{d P}{d t}=\left[\frac{\% P_{a l}}{100} I_{A}-\frac{\% P_{p e z}}{100} \frac{d B m}{d t}+f_{\text {otros }}\right]\left(1-R_{\text {pez }}\right)-\frac{P}{T} \\
= & {\left[\left(\frac{\% P_{a l}}{100}-\frac{\% P_{p e z}}{100} \frac{1}{T C A}\right) I_{A}+f_{\text {otros }}\right]\left(1-R_{\text {pez }}\right)-\frac{P}{T} }
\end{aligned}
$$

La concentración de fósforo $[\mathrm{P}](\mathrm{t})$ se obtiene con $\mathrm{P}(\mathrm{t})$ / $\mathrm{V}$, resolviendo la ecuación (11) para $\mathrm{P}$, con IA constante, de manera que la ecuación para $\mathrm{P}$ se desacopla de la ecuación de $\mathrm{Bm}$, con lo que $\mathrm{P}(\mathrm{t})$ tiende a un valor de equilibrio Peq para t "infinito". Partiendo del hecho de que el fósforo total es la contribución de dos tipos de fósforo, se obtiene la ecuación (12), que tiene por resultado:

$$
=\left[f_{\text {otros }}\left(1-R_{\text {pez }}\right)-\frac{P_{o t r o s}}{T}\right]+\left[f_{\text {exc }}\left(1-R_{p e z}\right)-\frac{P_{\text {exc }}}{T}\right]
$$

Donde Potros es el fósforo disuelto en la columna de agua proveniente de fuentes distintas a la actividad piscícola (kg de fósforo), y el valor medido para $\mathrm{P}$ corresponde a Pinicial e igual a Potros(0), ya que la contribución de Pexc es nula en ausencia de piscicultura. Se define una tasa de ingreso fexc, que es la contribución del alimento suministrado al fósforo del sistema.

En cuanto al fósforo de otras fuentes en la ecuación (12), se puede estimar Potros, suponiendo que el sistema se encuentra en equilibrio, momentos previos al inicio de la actividad (entrada constante de fósforo en un tiempo suficientemente largo para asegurar el estado de equilibrio). Entonces, la tasa de cambio de Potros es nula, y se obtiene la siguiente ecuación (13):

$$
f_{\text {otros }}=\frac{P_{\text {inicial }}}{\left(1-R_{\text {pez }}\right) T}
$$

Este valor en la ecuación (13) se interpreta como la entrada de fósforo necesaria para igualar la salida que permita mantener constante la concentración de fósforo, en una situación sin actividad de piscicultura, situación que puede variar debido principalmente a las acciones de transporte por advección, difusión, reacción o degradación, en relación con su comportamiento fisicoquímico en el cuerpo de agua.

\subsection{Comportamiento fisicoquímico de un contaminante en un cuerpo de agua}

Cuando un contaminante es vertido en un cuerpo de agua, sufre un cambio de concentración debido principalmente a tres acciones: transporte por advección, difusión, reacción o degradación, por lo que todo modelo matemático de simulación debe basarse en la evaluación de estas variables. [21]

La difusión consigue que la concentración de un contaminante varíe, principalmente con la profundidad, por lo que al realizar la modelación se debe considerar que la cantidad de agua y de contaminante que entra no varía con el tiempo.

La reacción es el cambio de concentración que sufre el contaminante por su interacción con los componentes químicos (especialmente el oxígeno) y biológicos (disminución o crecimiento las especies) del agua. Un ejemplo lo constituye la DBO; aquí el oxígeno reacciona con la materia orgánica (contaminante), y la transforma en dióxido de carbono, hidrógeno y agua [21]. 
Diseño de un módulo electrónico para la crianza automatizada de peces mediante modelamiento matemático multiparamétrico que simule las condiciones básicas necesarias para la crianza, en estanques artificiales en función de parámetros fisicoquímicos

Según factores [21], todo cuerpo de agua tiene una capacidad asimilativa que reduce la contaminación a través del tiempo, si no se producen nuevas cargas contaminantes. La consideración más importante para determinarla es la habilidad que presenta para mantener una concentración de oxígeno disuelto, afectada por:

Temperatura: el aumento reduce el valor de saturación de OD, e incrementa la degradación de la materia orgánica.

- $\quad$ El oxígeno que consumen los microorganismos para degradar la materia orgánica (DBO).

- Reaireación. Es la recuperación de oxigeno que realiza el cuerpo de agua para compensar el consumo generado por la DBO, la cual se puede realizar, por ejemplo, a través de la fotosíntesis de las plantas acuáticas.

- $\quad$ Salinidad. La presencia de sal en el agua hace que disminuya la capacidad para retener el oxígeno disuelto.

Es así que cuando se presentan descargas de contaminantes que se degradan con el tiempo, la concentración del contaminante al cabo de $t$ años se puede determinar mediante la expresión [21], como se observa en la ecuación (14):

$$
C(t)=\frac{W}{Q+V * K}\left[1-e^{-t(k+1 / T)}\right]+\left[C_{0} e^{-t(K+1 / T)}\right]
$$

Cuando se trata de sustancias conservativas, es decir, que no se degradan con el tiempo, la velocidad de degradación $\mathrm{K}$ es igual a cero [21].

Los parámetros que definen la ecuación de [21] son los siguientes; $\mathrm{Q}=\mathrm{Qe}+\mathrm{Qd}=$ Caudal de salida del sistema $=$ Caudal de entrada + Caudal de descarga neto (corriente, lluvia, evaporación); $\mathrm{W}=\mathrm{QeCe}+\mathrm{QdCd}=$ Masa total del contaminante que entra, $\mathrm{Ce}=$ Concentración de entrada; $\mathrm{Cd}=$ Concentración de entrada o salida de una descarga, $\mathrm{Cm}=$ Concentración resultante de la mezcla; $\mathrm{T}=$ Tiempo de retención hidráulica (mide la relación en horas entre el caudal y el volumen del depósito de aireación); $\mathrm{K}=$ Velocidad de degradación del contaminante, y Co = Concentración inicial de contaminante. A través de los años la concentración que existe, $\mathrm{Cm}$, va igualándose a la concentración que entra. Se dice entonces que se ha alcanzado una concentración de equilibrio, lograda al cabo de tres años.

Los nutrientes que contribuyen al proceso de eutroficación o de enriquecimiento con nutrientes y, por tanto, de sobreproducción de vegetación acuática, especialmente algas, son el nitrógeno y el fósforo. Sin embargo, la remoción del nitrógeno no es suficiente para realizar su control, porque existen algas que toman el nitrógeno que requieren de la atmósfera. Es así que la remoción de fósforo es más efectiva para realizar su control [21].

Teniendo en cuenta que el coeficiente de retención de fósforo $\mathrm{R}$ es mayor cuando la profundidad es baja, pues el fósforo en estos casos es retenido principalmente por sedimentación, donde a bajas profundidades la sedimentación es mayor; el tiempo te en el cual la concentración del estanque se iguala a la concentración que trae la corriente que lo alimenta; se puede calcular como [21], según se aprecia en la ecuación (15):

$t_{e}=\frac{4.6}{K+(1 / T)}=\frac{4.6}{R_{p}+(1 / T)}$

Sea P0 la concentración inicial, al cabo de t años, habrá una concentración Pt que se calcula, como se observa en la ecuación (16):

$$
P_{t}=\frac{W}{Q}\left[1-e^{-\frac{t}{T}}\left(1-P_{o}\right)\right]
$$

\section{Resultados}

\subsection{Medición de los parámetros indispensables}

\subsubsection{Pruebas de campo a los estanques artificiales para la producción de peces}

Existen en el mercado diferentes kits de medición para el análisis de los parámetros más importantes en piscicultura, pero la mayoría oscilan entre los 2’000.000 a los $10^{\prime} 000.000$ de pesos; razones por las cuales pequeños y medianos productores de la región de Guanentá no invierten en este tipo de tecnología, haciendo uso de labores manuales que les demandan tiempo, desplazamientos (nocturnos) y herramientas de monitoreo tediosas, por la cantidad de información.

Es por esto que después de una serie de visitas tanto a pequeños como medianos productores de la región Guanentá, en Santander, se realiza un exhaustivo estudio sobre los parámetros tenidos en cuenta comúnmente por los piscicultores, que se deciden por simplicidad, así como por reducción de costos, optimización del proceso reduciendo el número de sondas a dos (una por cada

factor), en función de la relevancia de cada variable en el proceso, sus múltiples efectos negativos para los peces y por su correlación con las demás variables. 
Seleccionando entonces, el $\mathrm{pH}$ por parte de las variables químicas y la temperatura por parte de las físicas, pues son variables relativamente sencillas de medir, así como de controlar, a un muy bajo costo.

\subsubsection{Medición de la temperatura en el cuerpo de agua}

La temperatura de los peces es aproximadamente la misma que la del cuerpo de agua, por tanto, es importante caracterizar sus fluctuaciones en diferentes estanques, horas del día, así como condiciones climáticas, para obtener un mejor modelo matemático.

Para ello se utiliza una sonda con un sensor DS18B20 integrado. Este es un termómetro digital de alta precisión, para el cual no existe degradación de la señal, y debido a que contiene un número de serie único, pueden existir varios en el mismo bus, lo cual permite colocar varios sensores en diferentes lugares.

Con este, se monitorean durante varios días diferentes topologías de estanques en diferentes condiciones ambientales, con Arduino como datalogger, lo que permite exportar estos datos a un archivo de Excel como el presentado en la tabla 3, en lapsos de 24 a 72 horas, para un máximo de 3 días por periodo de prueba. Esto con el propósito de obtener un modelo bastante aproximado a las múltiples condiciones, e independiente del tipo de estanque.

Tabla 3. Histórico diario de la temperatura del agua en los diferentes tipos de estanques.
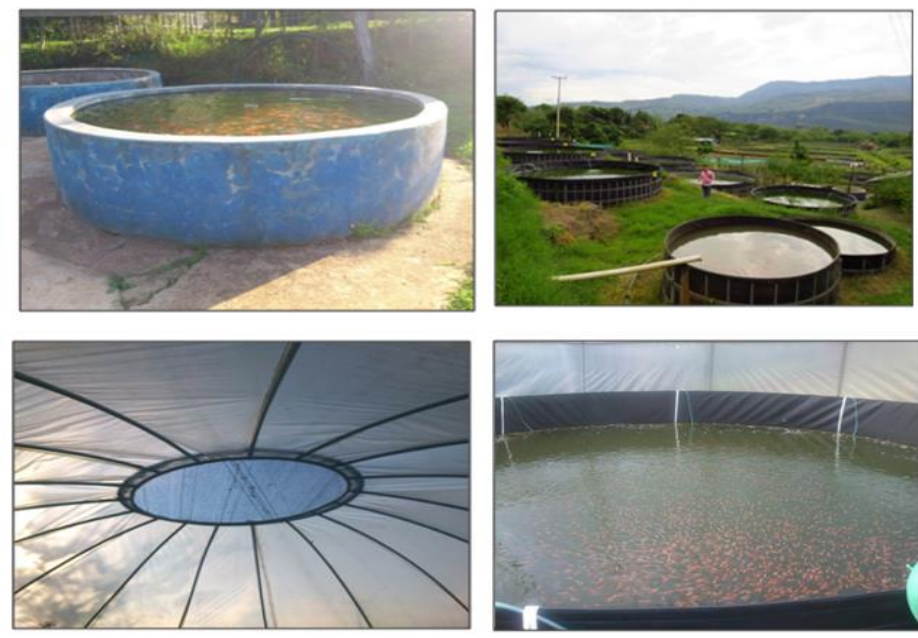

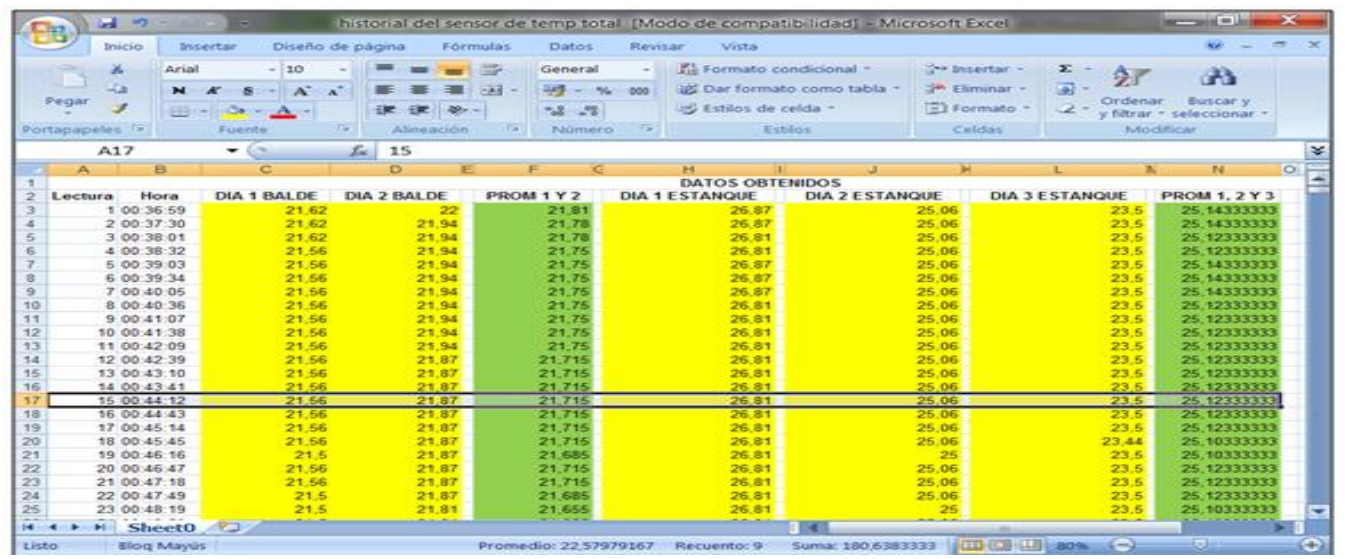

Fuente: elaboración propia.

A partir de estos resultados se obtuvo la curva de tendencia que describe el comportamiento del sistema independientemente de las condiciones (día, hora, estanque, volumen de agua, etc.), las cuales se ajustan satisfactoriamente a los resultados esperados según las investigaciones realizadas sobre el estado de la técnica.

Las pruebas se realizan con o sin oxigenación en el cuerpo de agua. Asimismo, reproduciendo 
Diseño de un módulo electrónico para la crianza automatizada de peces mediante modelamiento matemático multiparamétrico que simule las condiciones básicas necesarias para la crianza, en estanques artificiales en función de parámetros fisicoquímicos

periódicamente para cada estanque mínimo dos veces, con el propósito de obtener un promedio. Es importante aclarar que el estudio considera únicamente la implementación para estanques de geomembrana en domos "herméticos" con condiciones "aisladas" de las condiciones ambientales externas, razón por la cual la temperatura media del agua se hace mucho más estable, notando que cuanto más grande es el estanque, más estable es la temperatura. También que cuanto más pequeño es el estanque, se debe medir la temperatura más a menudo, siendo el mejor momento poco después de la salida del sol, entre las 6 y las 8 horas (temperatura mínima), y poco después de mediodía, entre las 12 y las 14 horas (temperatura máxima).

En estanques de menos de 1,25 metros de profundidad, es suficiente medir la temperatura del agua únicamente en su superficie, pero en estanques cuya profundidad sea superior, es preferible medir la temperatura cerca del fondo.

\subsubsection{Medición del pH en el cuerpo de agua}

El agua cuyo $\mathrm{pH}$ varía entre 6,5 a 8,5 (amanecer), en general las más apropiadas para la producción de peces en estanques. La mayor parte de estos peces muere en aguas con $\mathrm{pH}$ inferior a 4,5 o superior a 10,5 , y esto permite corregir el $\mathrm{pH}$ fuera de estos intervalos, para garantizar la no disminución en la producción de peces. El pH normalmente es menor en la madrugada, y es mayor en la tarde, cuando el uso de $\mathrm{CO} 2$ es alto, por lo que los niveles de OD son menores.

Para corroborar estas condiciones, se implementa el sensor analógico SKU: SEN0161, práctico por su fácil integración con Arduino. Además, el anillo en su membrana es de teflón, por lo que es adecuado para la detección a largo plazo, con una respuesta rápida, una excelente estabilidad térmica, buena reproducibilidad e inmunidad a la hidrólisis.

Para la recolección de los datos, se implementa el diagrama proporcionado por el fabricante presentado en la figura 1 (izquierda), instalándose una expansión Shield $\mathrm{SD}$, en la cual se almacenan los datos recolectados, los cuales además se pueden visualizar en tiempo real. Se establece una frecuencia de muestreo de 0,2 muestras por segundo, es decir, que el valor de $\mathrm{pH}$ es censado cada 5 segundos. Para estas pruebas es necesario que la sonda se encuentre sumergida durante alrededor de un minuto para estabilizar la lectura.
En esta fase se verifica además el sensor frente a un instrumento de referencia. Para ello se visita la Planta de Acueducto y Alcantarillado del Municipio de San Gil Acuasán, a fin de probar con soluciones con valores de $\mathrm{pH}$ preestablecidos, contrarrestando las lecturas con un instrumento patrón de tipo industrial de "mejores" características con mayor precisión, realizando un total de 22 a 14 muestras, lo que da como resultado un error promedio de $37,6 \%$, según se puede observar en la figura 1.
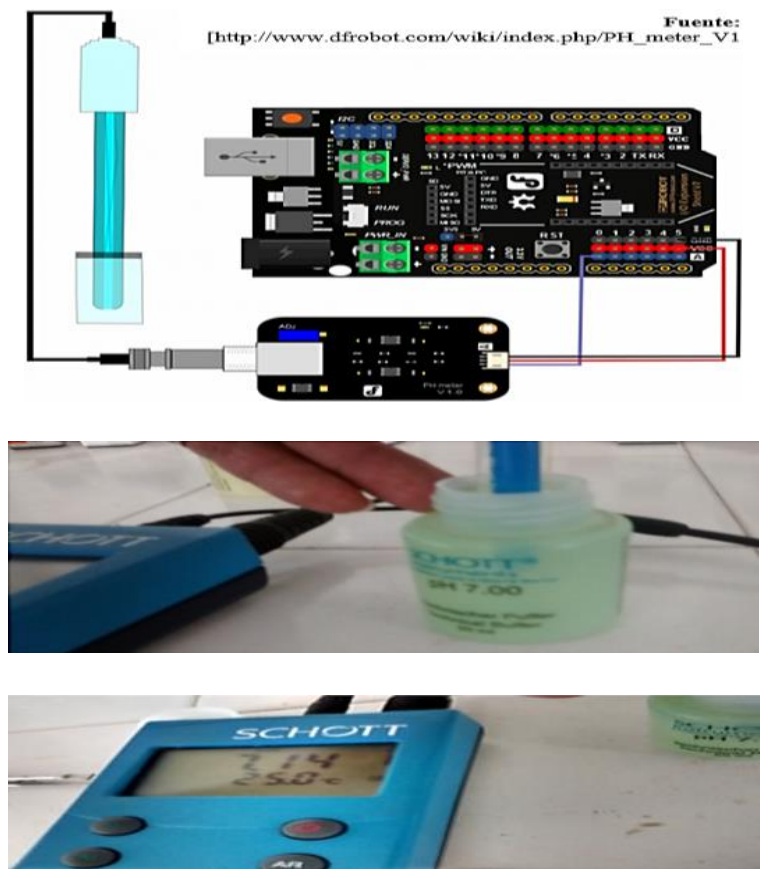

Figura 1. Diagrama de Conexión para los resultados con la solución de pH e instrumento patrón. Fuente: elaboración propia.

Se obtiene que la exactitud promedio del sensor es del $51,2 \%$; una precisión del $24,36 \%$, equivalente a una desviación promedio de 2,5. Este dato es tenido en cuenta al momento de programar, mapear o calibrar el sensor.

Se hacen pruebas de 2 a 8 horas durante 1 a 4 días, en diferentes volúmenes de agua, registrando además el estado del clima, según se puede observar en la figura 2 en el pantallazo de la interfaz de "Arduino Data Receptor", al momento de comenzar el primer ciclo de 6 horas junto con una vista a la hoja de datos de Excel. 
San Gil

Tormenta eléctrica Viento a $4 \mathrm{mph}$

Precip. 100\%
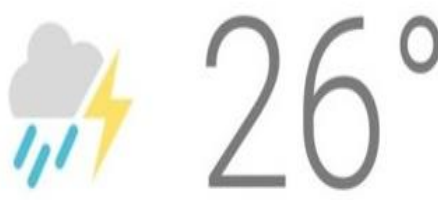

)

JUE. VIE. SÁB. DOM. LUN.

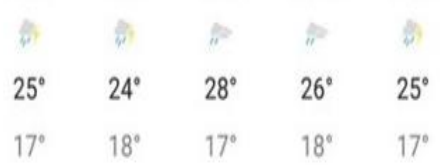

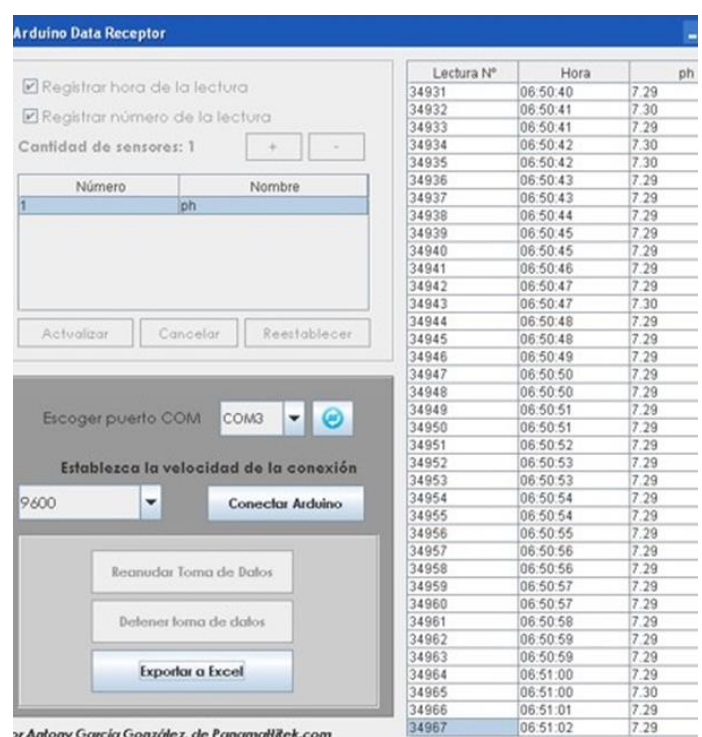

H. Díaz-López, Y. Vargas-Gómez

\begin{tabular}{|c|c|c|}
\hline & B & C \\
\hline 1 & Datos Obt, nidos & \\
\hline 2 & Lectura $\mathrm{N}^{\circ}$ Hora & ph \\
\hline 3 & $100: 58: 07$ & 7.23 \\
\hline 4 & $200: 58: 08$ & 7,23 \\
\hline 5 & $300: 58: 09$ & 7,23 \\
\hline 6 & $400: 58: 09$ & 7.23 \\
\hline 7 & $500: 58: 10$ & 7,23 \\
\hline 8 & $600: 58: 10$ & 7,23 \\
\hline 9 & $700: 58: 11$ & 7,24 \\
\hline 10 & $800: 58: 12$ & 7,23 \\
\hline 11 & $900: 58: 12$ & 7,24 \\
\hline 12 & $10 \quad 00: 58: 13$ & 7,23 \\
\hline 13 & $1100: 58: 13$ & 7.23 \\
\hline 14 & $1200: 58: 14$ & 7,23 \\
\hline 15 & $1300: 58: 15$ & 7,24 \\
\hline 16 & $1400: 58: 15$ & 7.23 \\
\hline 17 & $1500: 58: 16$ & 7,23 \\
\hline 18 & $1600: 58: 16$ & 7,23 \\
\hline 19 & $1700: 58: 17$ & 7.23 \\
\hline 20 & $1800: 58: 18$ & 7,24 \\
\hline 21 & $1900: 58: 18$ & 7,23 \\
\hline 22 & $2000: 58: 19$ & 7.23 \\
\hline 23 & $2100.58: 19$ & 7,23 \\
\hline 24 & $2200: 58: 20$ & 7,23 \\
\hline 25 & $2300: 58: 21$ & 7,23 \\
\hline 26 & $2400.58: 21$ & 7,23 \\
\hline 27 & $2500: 58: 22$ & 7,23 \\
\hline 28 & $26 \quad 00.58: 22$ & 7,23 \\
\hline 29 & $2700: 58: 23$ & 7,23 \\
\hline 30 & $2800: 58: 24$ & 7.24 \\
\hline
\end{tabular}

Figura 2. Estado de la prueba 1 del día 1 entregado por la interfaz de ARDUINO a la hoja de Excel. Fuente: elaboración propia.

La variación de la curva de $\mathrm{pH}$ en el estanque a lo largo de seis meses tiene un comportamiento promedio en un rango comprendido entre el 5,1 a 5,7. Cabe destacar que los requerimientos de $\mathrm{pH}$ para este proceso objeto de estudio se encuentran enmarcados entre 5,0 a 5,5, mientras que los límites de control están en 4,5 a 6,0, según los referentes de investigación en el estado del arte.

Debido a esto resultados se puede observar que el $\mathrm{pH}$ varía durante el día, que es mejor medirlo a intervalos regulares de dos o tres horas, desde la salida del sol hasta que el sol se pone, por lo que al amanecer el pH es más bajo, comparado con el final de la tarde donde alcanza su máximo, fluctuación que varía en intensidad cuando el estanque es más productivo.

En conclusión, es importante conocer no sólo el valor del $\mathrm{pH}$, sino la estabilidad o consistencia del mismo, incluso aunque no sea exactamente el $\mathrm{pH}$ que el pez necesite, pues los cambios bruscos son perjudiciales para el cultivo. Por lo general, los peces pueden sobrevivir en un rango de niveles de $\mathrm{pH}$, siempre y cuando esté cerca del óptimo y se mantenga regulado.

\subsection{Caracterización e identificación del sistema usando Matlab}

El modelo fue aplicado a seis cuerpos de agua con distinto número de peces, proporcional al volumen de agua, para comparar o valorar su veracidad en casos reales, teniendo en cuenta que, en este modelo, se han integrado la gran mayoría de procesos que ocurren en este tipo de entornos, con base en los modelos estandarizados, obteniendo los resultados que se pueden observar en la figura 3 , con:
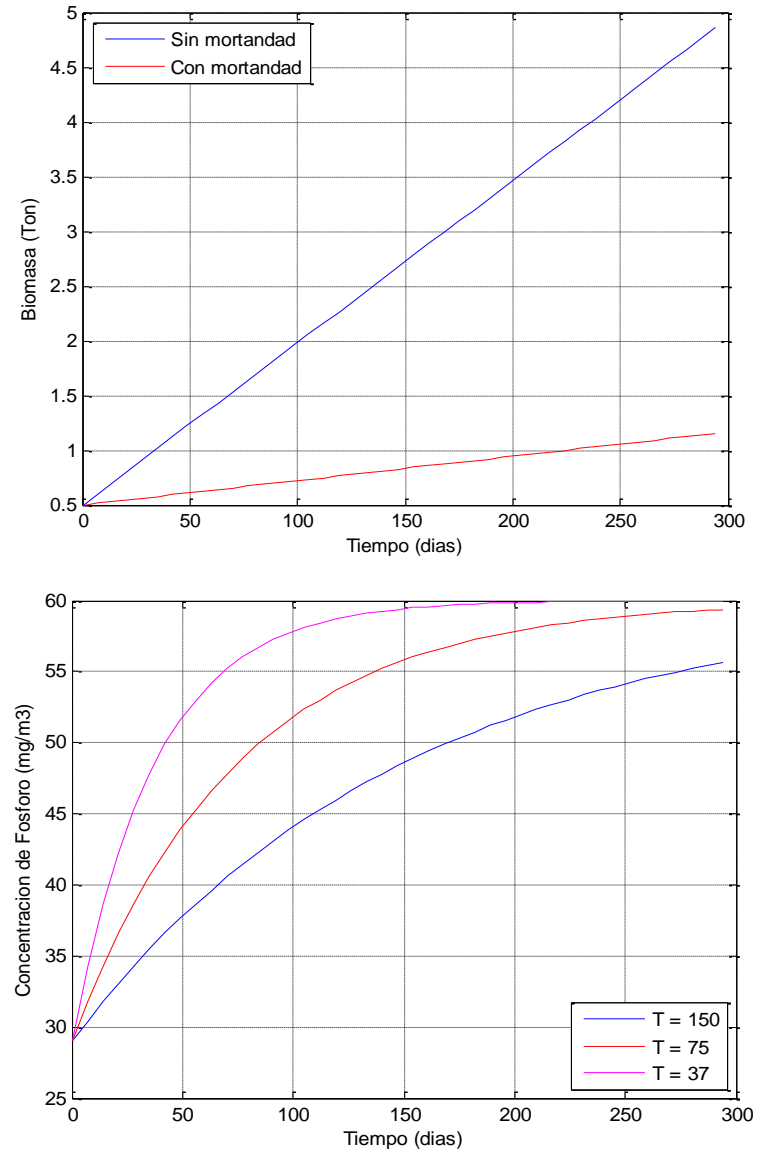

Figura 3. Resultados obtenidos para la biomasa de peces cultivados junto con la concentración de fósforo. Fuente: elaboración propia.

En la figura 3, se observa un crecimiento lineal de la biomasa Bm, debido a que IA se supone constante, sin tener en cuenta la mortalidad, estimando que, para 
Diseño de un módulo electrónico para la crianza automatizada de peces mediante modelamiento matemático multiparamétrico que simule las condiciones básicas necesarias para la crianza, en estanques artificiales en función de parámetros fisicoquímicos

obtener en un lapso de 180 a 270 días, peces de 450 gr, con una mortandad del $15 \%, I_{A 1} * T C A_{2}=0.15 * I_{A 2} *$ TC $A_{1}$

Asimismo, se observa un aumento en la concentración de fósforo $\mathrm{P}$, debido al alimento suministrado como por otras fuentes; desde un valor inicial Pinicial hasta un valor de equilibrio Pfinal (Peq), alcanzando el valor recomendado, exclusivamente con un tiempo de descarga o de "retención hidráulica" (relación en horas entre el caudal y el volumen del depósito de aireación) de 37 a 75 días, es decir, cada dos a tres meses.

Para proceder a diseñar el sistema de control, se obtiene el modelo representativo de la dinámica de la planta que reproduce con mayor exactitud el comportamiento de este proceso, pues uno de los problemas constantes es la limitante de no poder visitar regularmente las diferentes granjas para esta toma de datos ni poder hacer cambios a los mismos sin experimentar físicamente sobre los estanques, lo que permite, a partir de este, predecir su comportamiento, trabajar desde la comodidad del laboratorio, y favorecer además el no estrés de los peces por manipulación al cuerpo de agua.

La caracterización se realiza mediante la Toolbox de Matlab para la Identificación de Sistemas, a partir de los datos recolectados ante diversos cambios, y obtener varias funciones de transferencia que representan la dinámica de la planta, según se puede observar en la figura 4:

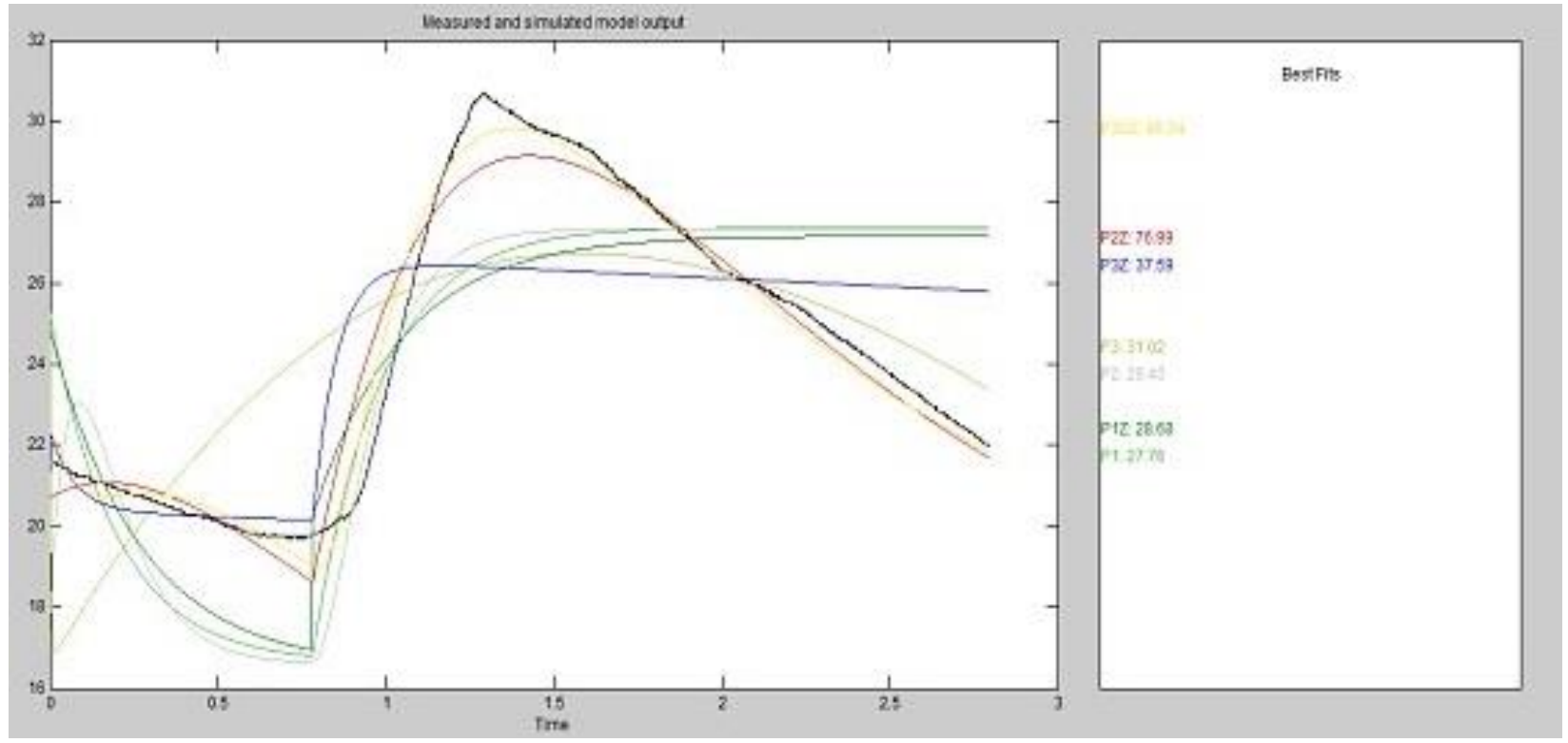

Figura 4. Resultados presentados en la Ventana “Process Models". Fuente: elaboración propia.

La respuesta en amarillo tiene un best fit o grado de compatibilidad más alto ( $85 \%)$, de modo que es la que mejor se adaptó a los datos de entrada-salida del proceso real. No obstante, por simplicidad de diseño (un cero dos polos), y con un $77 \%$ de compatibilidad, se opta por trabajar con el modelo de la función de transferencia representado por la gráfica en rojo, P2Z, cuya función de transferencias está dada por ecuación (17):

$$
\frac{0.588(1+3.646 s)}{(1+0.690 s)(1+0.692 s)}
$$

A continuación, se obtiene la función de transferencia correspondiente a los valores de $\mathrm{pH}$ entregados por la sonda, que caracteriza al sensor dentro del proceso, así como se hizo con la temperatura. De la misma forma, se obtienen las constantes de las demás funciones de transferencia, gracias al análisis correlacionado de la información obtenida, para finalmente, proceder a analizar la totalidad en conjunto con el sistema de control desarrollado.

Posteriormente, se obtienen las especificaciones de desempeño dada la dinámica de la planta, y se concluye, para el caso de la temperatura, por ejemplo, en un set point promedio de $29^{\circ} \mathrm{C}$, lo que permite variaciones menores a los $5^{\circ} \mathrm{C}$ (ideal), pues se sabe que cuando se presentan cambios repentinos mayores en la temperatura

del agua, el pez puede llegar a estresarse, o en el peor de los casos, puede llegar a morir. En el siguiente paso, se observan las respuestas parciales junto con la total del 
264 RUIII REVISTA UIS

sistema, y se verifica que se cumplen las restricciones preestablecidas para el régimen permanente, simulando el modelo en respuesta a diversas señales.

En la selección del controlador, se analiza el efecto de las distintas acciones de control, dadas las correspondencias preestablecidas en un comienzo, que se adaptan mejor a las condiciones ya instaladas en el escenario de pruebas final, debido a la reducción en los costos de implementación, al consumo de energía, así como factores importantes en el sistema como, el nivel de integración, visualización, circuito de potencia, entre otros.

\subsection{Integración final del sistema}

Se procede entonces a construir un prototipo físico de apoyo al dispositivo de control dinámico, implementado con un PLC Siemens Logo, el cual permite que la motobomba funcione en la menor cantidad de tiempo posible, lo que reduce principalmente el costo por consumo de energía, y hace más eficiente el proceso, pues en los casos en los que el PLC detecta algún tipo de fallo en el sensor, el accionamiento cambia automáticamente mediante el programador horario, según los periodos de tiempo más críticos del día para cada una de las diferentes variables, según la tabla 2; esto para dar respaldo al sistema.

Mediante estas condiciones se establece una reducción en el tiempo de marcha de la motobomba mayor al $40 \%$ del valor inicial, proporcional a una reducción igual en los costos de operación derivados de esta. Se reduce entonces la solución en cuanto a complejidad, costo de implementación, flexibilidad ante nuevas condiciones de operación, necesidad de implementar módulos adicionales de control, potencia, sensado o monitoreo, entre otras.

El sistema ha sido distribuido en dos tableros separados por parte electrónica (sensores $\rightarrow$ microcontrolador) y parte eléctrica (actuadores $\rightarrow$ PLC), comunicados entre sí. Esto, además de por diseño, por facilidad de uso, mantenimiento, manejo de señales, entre otros, pero sin inhibir la autonomía del control al PLC. Ese controlador adicional muestra información sobre los valores máximos y mínimos de cada una de las variables permanentemente en el estanque, a través de un LCD, como se puede apreciar en la figura 5.

\section{Conclusiones}

Según los promedios de la información dada por algunos fabricantes en materia de instrumentación, cada variable tiene un costo promedio de 2'000.000 de pesos, razón por la cual se propone trabajar un número reducido de
H. Díaz-López, Y. Vargas-Gómez

elementos, intentando obtener un modelo que permita estimar o correlacionar unas con otras, para reducir los costos de implementación, de aquí la importancia de trabajar con un modelo matemático que represente la dinámica del proceso, que permitió hacer pruebas en ausencia de ciertas variables sin interferir directamente en el proceso, es decir, sin alterar o comprometer el hábitat normal de los peces en los estanques de prueba.

Se llevó a cabo el diseño de un sistema de automatización, lo que ha favorecido el ahorro de energía, y ha establecido periodos de trabajo para los dispositivos de potencia durante el tiempo realmente necesario, a partir de las características de algunos de los parámetros representativos en la calidad del agua, con lo que se han controlado algunas de ellas en función de otras.

Es importante obtener la mayor cantidad de datos posibles para asegurar la suficiente información de la dinámica de cualquier sistema. De igual forma, es necesario validar diferentes modelos antes de escoger el(los) modelo(s) definitivo(s), para reducir la solución a una "mínima expresión" sin perder exactitud o precisión.

Calibrar cada elemento de sensado se hace indispensable, a través de algún tipo de solución estándar o instrumento patrón, que permitan, por ejemplo, a partir de la programación, mapear u obtener una mejor precisión en cada toma de medidas, a fin de mitigar el porcentaje de error final en cada una, según correspondan.

En el desarrollo del trabajo se analizaron específicamente cuatro modelos matemáticos, adaptados a la realidad de los procesos piscícolas en la región Guanentá, ajustando progresivamente el grado de dificultad de los mismos, al obtener una solución analítica, a través de la reducción de las ecuaciones propuestas por cada uno de los autores, con base en cada una de las suposiciones presentadas. Así se llega a un único modelo matemático que integra cada una de las mejoras, al tiempo que prioriza aquellas variables más representativas dentro del proceso, para permitir la reducción de la instrumentación requerida para el monitoreo de la calidad en el cuerpo de agua.

El régimen de alimentación moderadamente constante mejora la conversión del alimento en masa corporal, sin comprometer el crecimiento. Esto reduce las pérdidas de pienso, en redundancia con un ahorro sostenible del proceso; sin embargo, según el modelo, se debería utilizar junto con las tablas del cálculo de consumo de alimento, solamente de 3 a 5 veces durante todo el proceso, es decir, que se debe redefinir cada 60 a 90 días. 
Diseño de un módulo electrónico para la crianza automatizada de peces mediante modelamiento matemático multiparamétrico que simule las condiciones básicas necesarias para la crianza, en estanques artificiales en función de parámetros fisicoquímicos
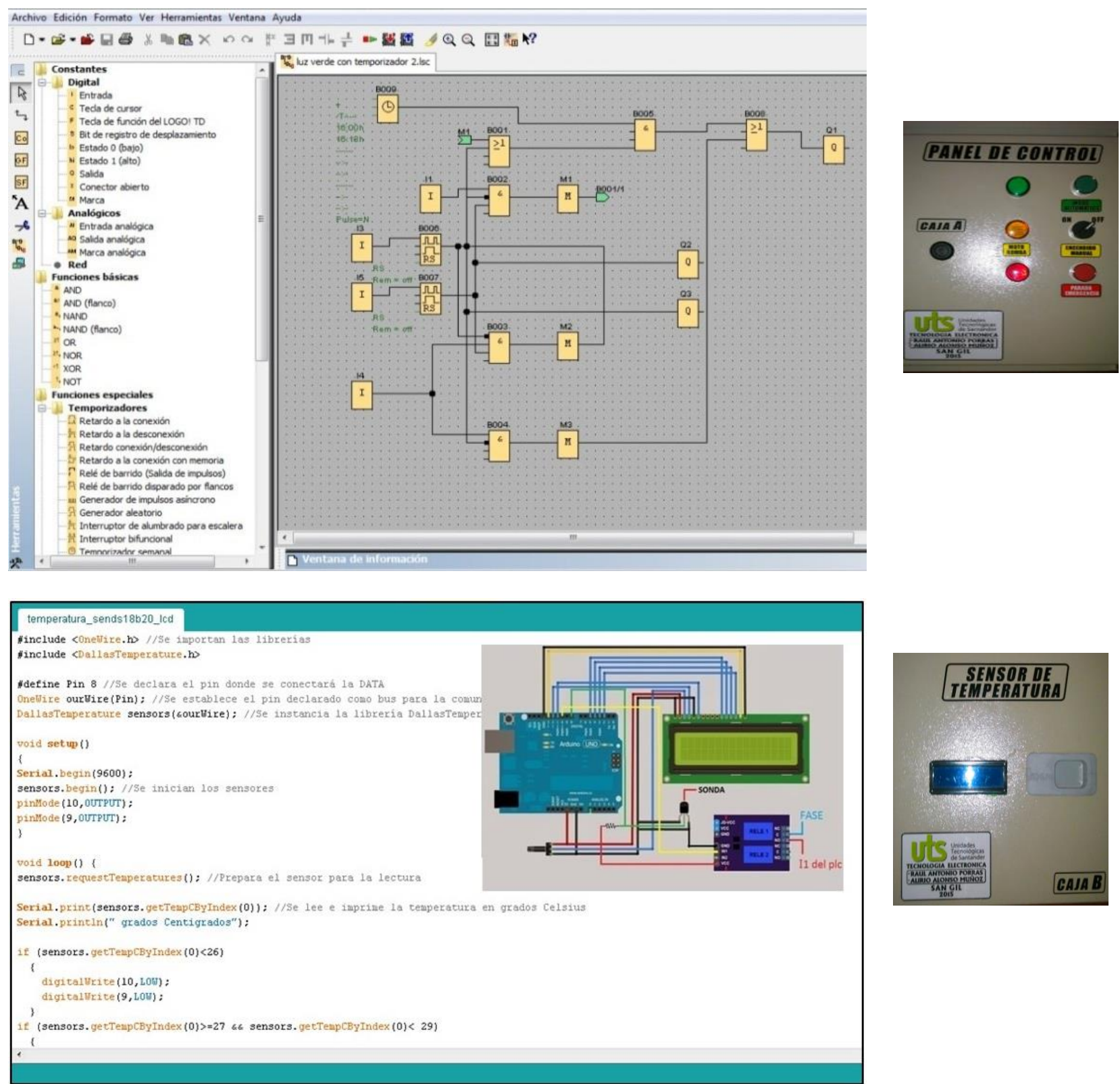

Figura 5. Cuadro general de conexiones del sistema usando el software de Siemens junto al Arduino. Fuente: elaboración propia.

Los individuos presentan perfiles de crecimiento influenciados por la época del año en la que se inicia el cultivo, induciendo patrones transitoriamente distintos en función de las condiciones climatológicas. Como consecuencia de esto, se debería comprobar en controles a corto plazo, su monitorización utilizando preferiblemente los parámetros TGC (Coeficiente de Crecimiento por Unidad Térmica) y FTGC (Coeficiente "Fototérmico" del Crecimiento), en vez de la SGR (Tasa Específica de Crecimiento), teniendo en cuenta que, dentro de los parámetros para la caracterización se incluye un modelo en función de la temperatura.
Se han descrito algunos de los modelos creados hasta la fecha del presente estudio. Se han indicado brevemente las mejoras o aportaciones en cada uno de ellos, y se ha comprobado su veracidad con los modelos propuestos, probados en seis casos experimentales reales. Se ha estudiado el crecimiento de una misma especie, en diferentes volúmenes de agua, así como su capacidad de producir biomasa y de consumir nutrientes en un lapso de seis a nueve meses (180 a 270 días), periodo recomendado para el ciclo de producción en general, pero el cual dependerá de la cantidad de animales sembrados, la temperatura y la cantidad de agua. 
El modelo permite simular escenarios con IA o TCA variable, de acuerdo con la edad del pez (semanas) medida desde el inicio de la actividad piscícola, según valores promedios obtenidos de las tablas para el cálculo de consumo de alimento, así como de la biomasa que se va a producir, en función de la escala del proyecto productivo de piscicultura, todo sin necesidad de medir más parámetros que los necesarios.

Los resultados obtenidos muestran las ventajas de los modelos matemáticos, al permitir, a través de su capacidad predictiva, estimar la dinámica de múltiples variables durante el tiempo de cosecha, a pesar de los cambios en muchas de ellas. Esto ha permitido establecer mediante ingeniería inversa las condiciones iniciales que posibiliten optimizar la productividad, indistintamente del tipo de proyecto piscícola, que aminore la afectación sobre el estado de los peces.

Pese a que todavía quedan muchos detalles que incorporar en el modelo, en vista de que a la fecha no se dispone de los datos experimentales diarios de variables como el carbono, nitrógeno, entre otros, no se puede realizar una comparativa de estas gráficas, así como de los errores poco significativos obtenidos, teniendo en cuenta la gran variabilidad que existe, así como las diversas fuentes de error que impiden que el proceso no se describa al $100 \%$; el nivel de precisión elevado obtenido en el estudio deja ver la gran capacidad de los modelos matemáticos de poder representar lo que ocurre en procesos reales, como en el caso particular de la piscicultura.

\section{Agradecimientos}

Este trabajo esta soportado por los aportes de los trabajos desarrollados por los estudiantes (A. Alonso, R. Porras, 2015), (B. Rincón, E. Caballero, 2017), además de las asignaturas de Señales y Sistemas, así como de la asignatura de Sistemas de Control Analógico, de las Unidades Tecnológicas de Santander (UTS) San Gil.

\section{Referencias}

[1]. A. Alonso, R. Porras, e Y. Vargas, "Prototipo de un sistema automatizado para la crianza de peces en estaques artificiales". Facultad de Ciencias Naturales e Ingenierías, Unidades Tecnológicas de Santander - UTS San Gil, 2015.

[2]. A. Regalado, E. Peralta, and C. González. "Cómo hacer un modelo matemático". Universidad del Mar, Campus Puerto Ángel, Instituto de Industrias - Instituto de Ecología. Temas de Ciencia y Tecnología vol. 12 No 35, págs. $9-18,2008$.
[3]. A. Stigebrandt, J. Aure, A. Ervik, P.K. Hansen, "Regulating the local environmental impact of intensive marine fish farming III. A model for estimation of the holding capacity in the Modelling-Ongrowing fish FarmMonitoring system". Aquaculture. 234:239-261, 2004.

[4]. Beveridge, M.C.M., "Piscicultura en jaulas y corrales. Modelos para calcular la capacidad de carga y las repercusiones en el ambiente". FAO Doc. Téc. Pesca, (255): 100 p., 1986. [En línea]. Disponible en: http:// www.fao.org/DOCREP/005/AD021S/AD021S00. HTM

[5]. B. Rincón, E. Caballero, e Y. Vargas, "Estudio de la tecnología en instrumentación piscícola empleada para el muestreo de la calidad del agua, con el fin de poder minimizar el número de variables implícitas en su proceso de medición". Facultad de Ciencias Naturales e Ingenierías, Unidades Tecnológicas de Santander - UTS San Gil, 2017.

[6]. C.J. Cromey, K. Nickell, D. Black, "Modelling the deposition and biological effects of waste solids from marine cage farms". Aquaculture. 214:211-239, 2002.

[7]. D. Cuesta-Parra, C. Velazco-Rincón, J. CastroPardo, "Evaluación ambiental asociada a los vertimientos de aguas residuales generados por una empresa de curtiembres en la cuenca del río Aburrá," Rev. UIS Ing., vol. 17, no. 2, pp. 141-152, 2018. Doi: https://doi.org/10.18273/revuin.v17n2-2018013

[8]. D. L. Bottom, D.J. Stouder, P.A. Bisson, R.J. Naiman, "To Till the water: a history of ideas in fisheries conservation. Pacific Salmon and Their Ecosystems: Status and Future Options". Ed. Chapman Hall (New York). p. 569-597, 1997.

[9]. D. Brigolin, R. Pastres, T.D. Nickell, C.J. Cromey, D.R. Aguilera, and P. Regnier, "Modelling the impact of aquaculture on early diagenetic processes in sea loch sediments". Marine Ecol. Progress Series. 388:63-80, 2009.

[10]. E. Mayer, H. Biomin, "Control de la calidad del agua de estanques para mejorar la producción de camarones y peces". [PDF]. Disponible en: https://cap.auburn.edu/blog/2012/05/control-de-lacalidad-del-agua-de-estanques-para-mejorar-laproduccion-de-camarones-y-peces/?lang=es.

[11]. E. García, I. Amaya, R. Correa, "Algoritmos de optimización en la estimación de propiedades termodinámicas en tiempo real durante el tratamiento térmico de materiales con microondas," Rev. UIS Ing., 
Diseño de un módulo electrónico para la crianza automatizada de peces mediante modelamiento matemático multiparamétrico que simule las condiciones básicas necesarias para la crianza, en estanques artificiales en función de parámetros fisicoquímicos

vol. 16, no. 2, pp. 129-140, 2017. Doi: https://doi.org/10.18273/revuin.v16n2-2017012

[12]. O. Gelvéz-Arocha, J. Quiroga-Mendez, D. BarajasMerchán, M. Gómez-Sarmiento, "Estudio experimental de las estrategias de control On-Off y control continuo en un sistema de refrigeración," Rev. UIS Ing., vol. 11, no. 1, pp. 73-82, 2012.

[13]. H.M. Buyukcapar, A. ALP, "The carrying capacity and suitability of the menzelet reservoir (Kahramanmaras- Turkey) for trout culture in terms of water quality". J. Appl. Sci. 6:2774-2778, 2006.

[14]. J. Cano, J. A. Luna, and Ch. A. Rivera, "Automatización de un Invernadero de Pez Tilapia". IPN, Escuela Superior de Ingeniería Mecánica y Eléctrica Unidad Culhuacán. México D.F, 2009.

[15]. J. Riascos, D. Díaz, L. Beltrán, F. Gutiérrez. "Modelo Dinámico para Estimar la Capacidad de Carga de Cuerpos de Agua con Piscicultura". Revista U.D.C.A Actualidad \& Divulgación Científica. ISSN 0123-4226, Vol.15 No.1. Bogotá, 2012.

[16]. N.C. Stickland, R.N. White, P.E. Mescall, A.R. Crook, and J.E. Thorpe. "The effect of temperature on myogenesis in embryonic development of the Atlantic salmon (Salmo salar, L.)" Anat. Embryol. 178:253-257, 1988.

[17]. R. Núñez, O. Pinzón, “Controlador robusto basado en la técnica QFT para convertidores DC-DC buck -boost como regulador de voltaje en generadores fotovoltaicos," Rev. UIS Ing., vol. 17, no. 1, pp. 243-250, 2018. Doi: https://doi.org/10.18273/revuin.v17n1-2018024.

[18]. P.J. Dillon, H.E. Evans, "A comparison of phosphorus retention in lakes determined from mass balance and sediment core calculations". Water Res. 27(4):659-668, 1993.

[19]. R.H. Findlay, L. Watling, "Prediction of benthic impact for salmon net-pens based on the balance of benthic oxygen supply and demand". Marine Ecol. Progress Series. 155:147-157, 1997.

[20]. S. Pulatsü, "The application of a phosphorus budget model estimating the carrying capacity of Kesikk. pr. Dam Lake). Turk. J. Vet. Anim. Sci. 27:1127- 1130, 2003.

[21]. W. Casas, C. Vargas, "Capitulo 5. Modelos matemáticos de simulación de calidad del agua en Colombia. Principios y aplicaciones". [PDF]. Disponible en:

http://biblovirtual.minambiente.gov.co:3000/DOCS/ME MORIA/MMA-0013/MMA-0013-CAPITULO6.pdf

[22]. Magill, S.H.; Thetmeyer, H.; Cromey, C.J. "Settling velocity of feacal pellets of gilthead sea bream (Sparusaurata L.) and sea bass (Dicentrarchus labrax) and sensitivity analysis using measured data in deposition model". Aquaculture. 251:295- 305, 2006.

[23]. Morales, V.V.; Morales, R. "Síntesis regional del desarrollo de la Acuicultura 1. América Latina y el Caribe. FAO Circular de Pesca No 1017/1, 2005.

[24]. Stickney, R.R. "How did we get into this mess? Junk science vs. real science". World Aquaculture.

$34: 71,2003$

[25]. Vollenweider, R.A. "The scientific basis of lake and stream eutrophication with particular reference to phosphorus and nitrogen as eutrophication factors". Tech. Rep. OECD, Paris, DAS/CSI 68. 27:1-182, 1968.

[26]. Vallentyne, J.R. "The algal bowl lakes and man". Dep. Environ. Fish. Mar. Serv., Ottawa. Misc. Publ. 22. 186p. 1974.

[27]. Sonzogni, W.C.; Chapra, S.C.; Armstrong, D.E.; Logan, T.J. "Bioavailability of phosphorus inputs to lakes". J. Environ. Qual. 11:555-562, 1982.

[28]. Canosa, A.; López, L.; Morales, D.; Martínez, P. "Línea Base Microbiológica para Ajuste del POPA (Plan de Ordenamiento Pesquero y acuícola) del Embalse de Betania. Informe Técnico. Instituto Colombiano de Desarrollo Rural Integral (Colombia). [PDF]. Disponible en:

http://www.huila.gov.co/documentos/P/POPABetaniaTe xto.pdf, 2008.

[29]. Kiely, G. "Fundamentos, Entornos Tecnologías y Sistemas de Gestión", Ingeniería Ambiental. McGrawHill, 1999.

[30]. Jover Cerdá, M. "Estimación del crecimiento, tasa de alimentación y Producción de desechos en piscicultura mediante un modelo Bioenergético". Dpto. Ciencia Animal. Laboratorio de Acuicultura, Universidad Politécnica de Valencia. [PDF]. Disponible en: file:///C:/Users/usuario/Downloads/70-120-1-

SM\%20(1).pdf

[31]. Larsen, D.P.; Mercier, Y.H.T. "Phosphorus retention capacity of lakes". J. Fish. Res. Board Can. 33(8):1742-1750, 1976. 
[32]. E. Mayer, H. Biomin, "Control de la calidad del agua de estanques para mejorar la producción de camarones y peces". [En línea]. Disponible en: https://cap.auburn.edu/blog/2012/05/control-de-lacalidad-del-agua-de-estanques-para-mejorar-laproduccion-de-camarones-y-peces/?lang=es 